\title{
Methodology of using the Adjoint solver optimization tool during flow in the intercooler filling line to minimize pressure drop
}

\author{
Veronika Horová ${ }^{1}$, Marian Bojko ${ }^{1, *}$, and Josef Dobeš² \\ ${ }^{1}$ Department of Hydromechanics and Hydraulic Equipment, Faculty of Mechanical Engineering, VŠB-Technical University of Ostrava, \\ 17. listopadu 15, Ostrava Poruba, Czech Republic \\ ${ }^{2}$ Hanon Systems Autopal Services s.r.o., Luzicka 984/14, Novy Jicin, Czech Republic
}

\begin{abstract}
The paper deals with numerical modelling of the flow in the intercooler filling line by Adjoint solver to minimize pressure loss. The ANSYS Fluent software was used for the calculations. The basic flow calculation was performed in the first phase. Then the mathematical model with Adjoint solver optimization tool was defined. The numerical calculation was unstable and did not lead to a convergent solution, because of creation of vortexes. The mathematical model was simplified in the second phase. To suppress instabilities and vortices a dynamic viscosity of coolant was adjusted. The pressure gradients between inlet and outlet for unmodified geometry and for modified geometry were evaluated. The final evaluations of pressure drop changes were implemented for modified geometry with original dynamic viscosity of the coolant.
\end{abstract}

\section{Introduction}

The optimization of a design using numerical flow modelling is an important part of the designing process and following manufacturing process. This make unnecessary to create physical models for practical exploring. This saves time and especially saves finances.

It is necessary to create and define a suitable 3D model for optimization. The 3D model is solved numerically to obtain the necessary data. There are many optimization methods. The best known optimization method is the gradient-based method that works with a large number of design variables. The gradient-based method uses computational schemes such as finite difference scheme and adjoint scheme to solve. The adjoint method is more preferable because it is independent on the design variables and performs the calculation only once. This method is part of the ANSYS Fluent software (Adjoint Solver), which allows to get information about the sensitivity of a fluid system that is difficult to get in another way. The Adjoint Solver extends the analysis that is contained in the standard flow solver. The user must complete all necessary information when simulating using a standard flow solver. The information includes mesh, materials properties, physical model and boundary conditions. If the flow calculation converges, the standard solver will provide a dataset describing the flow. The application of the Adjoint Solver in ANSYS Fluent software is the content of the publications [1,2]. There is described the methodology of application of the optimization tool on the examples of flow in a duct in car climate system and in a flow passage of the check valve.
This paper describes the methodology of using Adjoint Solver applied on the intercooler entry connector used in the automotive industry.

The intercooler (Figure 1) is a technical device that serves to heat exchange in supercharged automobile engines. The filling line is used to connect the pressure energy source with the cooling plates.

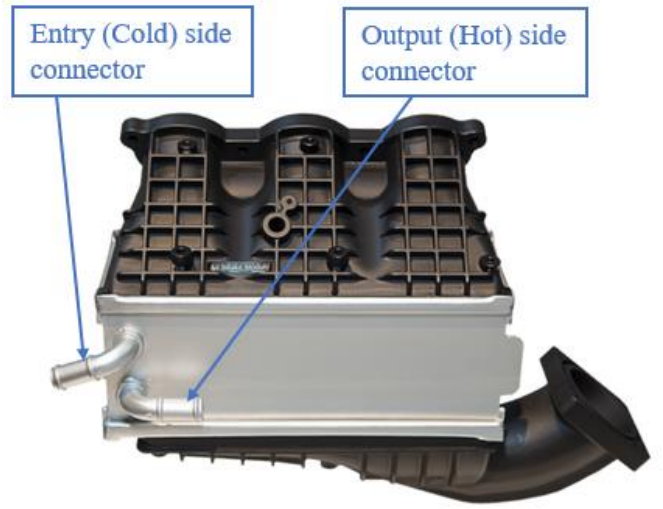

Fig. 1. Water charge air cooler

The ANSYS Fluent 18.2 software was used for the basic flow calculation and Adjoint Solver application. ANSYS Fluent software contains a discrete adjoint solver based on the discretized form of equations. A mathematical model of turbulence $k-\varepsilon$ RNG with wall function Enhanced Wall Treatment was used for shape optimization. By evaluating parameter $y+$ was verified that this mathematical model of turbulence is used correctly. The shape optimization was performed on the entry (cold) connector geometry.

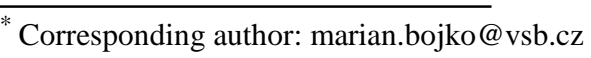


The use of the Adjoint Solver is unstable in applications where there are vortices or other instabilities in the flow field, which are created as a result of flow separation. This Adjoint Solver instability characteristic presents the issue of the solution for the intercooler entry connector that is content of this paper.

To prevent creating vortices, the dynamic viscosity of the coolant was adjusted. This change has led to convergent solution.

Currently, there is an increase in operating design parameters, which may be associated with the occurrence of cavitation [3] in certain geometry areas, such as narrowing. The use of the Adjoint Solver can predict these areas by evaluating pressure field.

\section{Mathematical model characteristics}

The mathematical model is defined by balance equations which describes the flow. They are continuity equation, Navier-Stokes equations, energy equation and more $[4,5]$. During the flow calculation on the entry connector geometry the energy equation was not used because the isothermal flow was defined.

There are several mathematical models of turbulence available in ANSYS Fluent software. The most used mathematical models of turbulence include dual-equation $k-\varepsilon$ mathematical model and $k-\omega$ mathematical model.

There are three major modifications for the $k-\varepsilon$ mathematical model of turbulence. They are $k-\varepsilon$ Standard, $k-\varepsilon$ RNG a $k-\varepsilon$ Realizable [4]. The $k-\varepsilon R N G$ mathematical model of turbulence is suitable for lower Reynolds numbers in existence of vortices and secondary flow. Reynold's critical number indicates the transition between the laminar and the turbulent flow and is defined by:

$$
\operatorname{Re}=\frac{v \cdot d}{v}
$$

$$
\begin{aligned}
& v \ldots \text { velocity }\left[\mathrm{m}^{-1} \mathrm{~s}^{-1}\right] \\
& d \ldots \text { hydraulic diameter }[\mathrm{m}] \\
& v \ldots \text { kinematic viscosity }\left[\mathrm{m}^{2} \cdot \mathrm{s}^{-1}\right]
\end{aligned}
$$

Dynamic viscosity is defined by:

$$
\eta=v \cdot \rho
$$

$$
\begin{aligned}
& v \ldots \text { kinematic viscosity }\left[\mathrm{m}^{2} \cdot \mathrm{s}^{-1}\right] \\
& \rho \ldots \text { density }\left[\mathrm{kg} \cdot \mathrm{m}^{-3}\right]
\end{aligned}
$$

Changing the dynamic viscosity reduced the Reynolds number and the flow regime was changed to laminar.

\section{Adjoint Solver}

Adjoint Solver [6] in ANSYS Fluent software is a tool that allows you to get information about the sensitivity of a fluid system that is difficult to get in another way. The Adjoint Solver performs a calculation that is very similar to the standard calculation. The difference is that scalar observation is selected before the calculation starts. If the convergence of the adjoint solution occurs, we obtain derivations of the observe magnitude relative to the position of each individual point located on the surface of the geometry. This determines the sensitivity of the monitored variable for a particular boundary condition setting. The final calculation of the adjoint task can be used as a pattern for the design change.

ANSYS Fluent software includes a discrete adjoint solver. By using a discrete adjoint solver, we get information for various problems, including the wall functions problem. The use of Adjoint Solver may be unstable, especially in applications where there are vortices or other instabilities in the flow field. Adjoint Solver stability can be problematic at high Reynolds numbers, especially when dealing with large numbers of cells and complex geometry. To overcome these problems, two stabilization schemes are available in ANSYS Fluent software (Spatial Scheme and Modal Scheme) [7].

\section{Mesh, Boundary conditions, Physical properties}

The mesh was created in the ANSYS Meshing. The mesh was densified near the wall according to the wall function. The suitability of the wall function was verified by evaluating the progress of parameter $y+$ [3] with additional adaptation. The result was a mesh with 4206535 elements.

The mathematical model characteristic is presented in Table 1. In Table 2 there are boundary conditions which were defined. In Table 3 there are physical properties of the coolant at $35^{\circ} \mathrm{C}$.

Table 1. Characteristics of defined mathematical model

\begin{tabular}{|l|l|}
\hline Model of geometry & $3 \mathrm{D}$ \\
\hline Incompressible flow & $\rho=$ constant \\
\hline Turbulent flow & $k-\varepsilon, k-\omega$ \\
\hline Isothermal flow & $T=$ constant $\left(35^{\circ} \mathrm{C}\right)$ \\
\hline Single-phase flow & \\
\hline
\end{tabular}

Table 2. Boundary conditions

\begin{tabular}{|l|l|}
\hline \multicolumn{2}{|c|}{ Inlet-mass flow inlet } \\
\hline Mass flow rate & $0.264166 \mathrm{~kg} \cdot \mathrm{s}^{-1}$ \\
\hline Turbulent intensity & $5 \%$ \\
\hline Hydraulic diameter & $0.01461 \mathrm{~m}$ \\
\hline \multicolumn{2}{|c|}{ Outlet-pressure outlet } \\
\hline Pressure & $0 \mathrm{~Pa}$ \\
\hline Turbulent intensity & $5.5 \%$ \\
\hline Hydraulic diameter & $0.02922 \mathrm{~m}$ \\
\hline
\end{tabular}

Table 3. Physical properties

\begin{tabular}{|l|l|}
\hline Density & $\rho=1045 \mathrm{~kg} \cdot \mathrm{m}^{-3}$ \\
\hline Dynamic viscosity & $\eta=0.0019 \mathrm{~Pa} \cdot \mathrm{s}$ \\
\hline
\end{tabular}

The calculation was unstable, when applying the physical properties from Table 3, therefore the dynamic 
viscosity was adjusted to $1 \mathrm{~Pa}$ s to ensure a stable and convergent calculation of adjoint variables. By adjusting the dynamic viscosity, the Reynold's number was reduced and more uniform flow character can be assumed. With respect to reduction of Reynold's number a $k-\varepsilon$ RNG with Enhanced Wall Treatment mathematical model of turbulence was used. This mathematical model of turbulence is suitable for lower Reynold's numbers.

\section{Mathematical model application-basic flow}

The basic flow was calculated in the first phase. The coolant was water-glycol in ratio 60/40 (60\% water, $40 \%$ glycol). Several mathematical models of turbulence have been tested. It was used $k-\varepsilon$ RNG with Enhanced Wall Treatment mathematical model of turbulence for evaluation, which is suitable for lower Reynolds numbers.

The progress of parameter $y+$ was evaluated. The value of a parameter $y+$ was about 1 which means that the wall function was appropriately selected. The calculation was applied to the entry connector geometry (Figure 2). Figure 2 shows the entry and output connector to intercooler. Inlet and outlet boundary conditions are marked here.

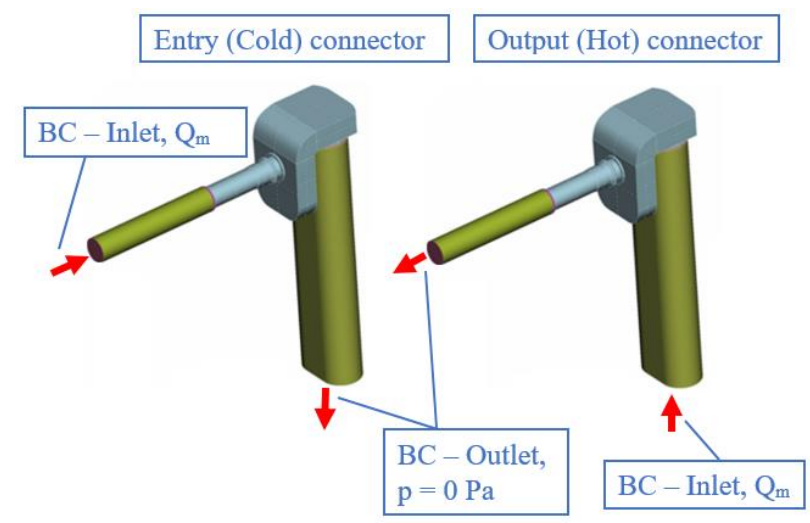

Fig. 2. Entry and output connector

In Figure 3 there is a comparison of the pressure drop values between inlet and outlet $\left(\Delta p_{I N, O U T}=\Delta p_{I N}-\Delta p_{\text {OUT }}\right)$ for several mathematical models of turbulence.

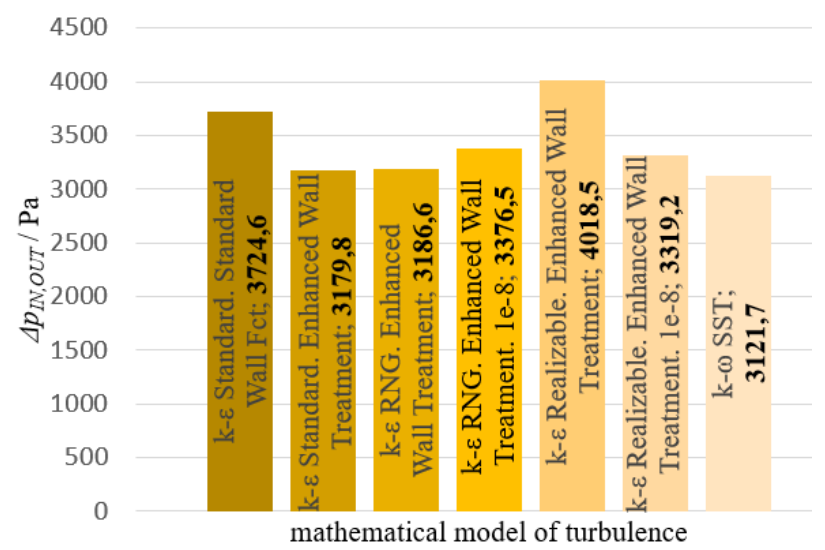

Fig. 3. Comparison of mathematical models of turbulence
The contours of total pressure (Figure 4) and contours of velocity magnitude (Figure 5) were evaluated in the longitudinal section of the computing area.

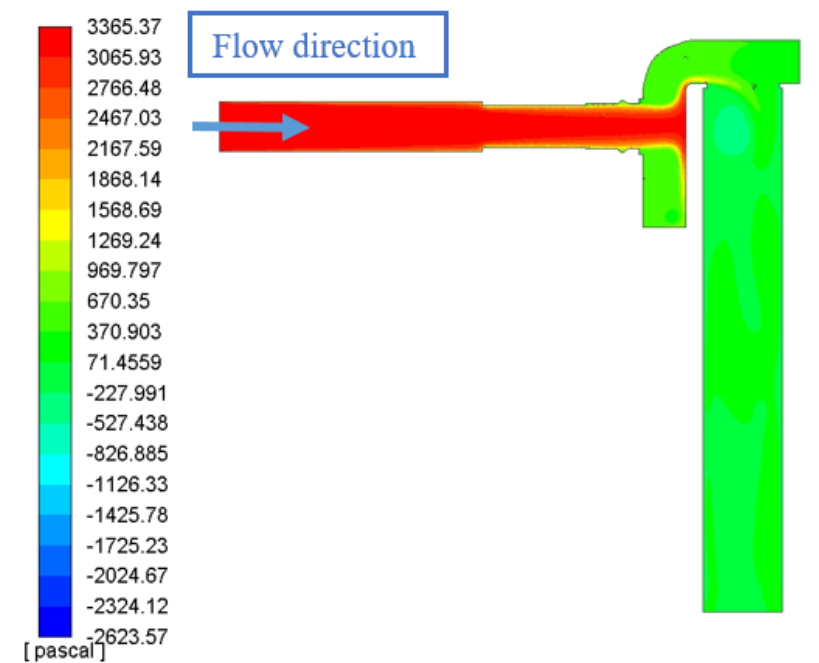

Fig. 4. Contours of Total pressure $p / \mathrm{Pa}$
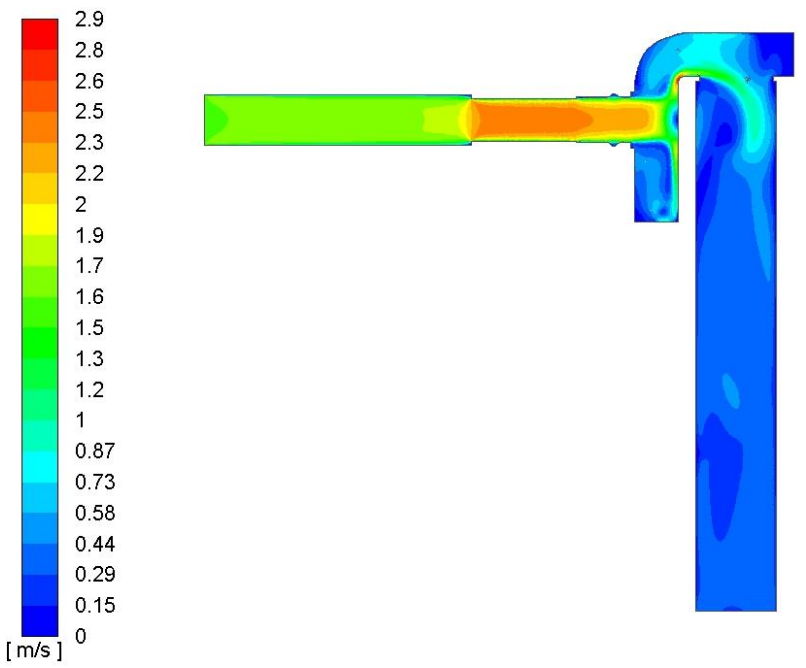

Fig. 5. Contours of Velocity Magnitude $v / \mathrm{m} / \mathrm{s}$

\section{Adjoint Solver application}

After a basic flow calculation, a shape optimization was performed for the $k-\varepsilon$ RNG Enhanced Wall Treatment mathematical model of turbulence on the entry connector geometry. There are vortices in the computing area and neither the use of stabilization schemes has not led to a convergent solution. The solution had a divergent character, which is evident from Figure 6. 


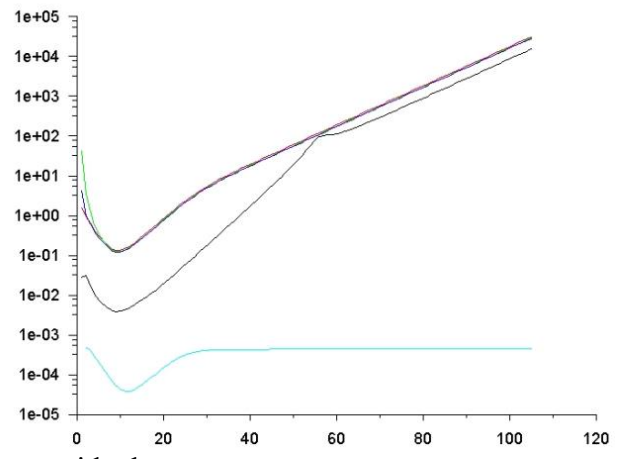

Fig. 6. Divergent residuals

There are several options to achieve convergent solution e.g. switch to a time-dependent solution, which Adjoint Solver does not allow. The dynamic viscosity of coolant was adjusted to $1 \mathrm{~Pa}$ s to avoid formation of vortices. By adjusting the dynamic viscosity, a more uniform flow character can be assumed. This provides stable and convergent calculation of adjoint variables. Then the optimization was performed. Any stabilization scheme was used during the calculation of adjoint variables.

The procedure of performing the shape optimization in the Adjoint Solver in individual steps is described in the next chapter.

\section{Optimization methodology}

The deformation region was defined only on the inlet part of the entry connector as shown in Figure 7.

Shape optimization steps:

- Pressure drop $\Delta p_{I N, O U T}$ evaluation

- Shape Sensitivity Magnitude evaluation

- Pressure drop expected change evaluation

- Modification of geometry

- Contours of Normal Optimal Displacement evaluation

- Display geometry change result

- The basic flow calculation

- The adjoint variables calculation

The pressure drop between inlet and outlet $\Delta p_{I N, O U T}$ for unmodified geometry was evaluated in the first step. Then the Shape Sensitivity Magnitude was evaluated (Figure 7), which is a prediction before the optimization of the geometry. It displays areas where the pressure drop is most sensitive to shape of geometry.
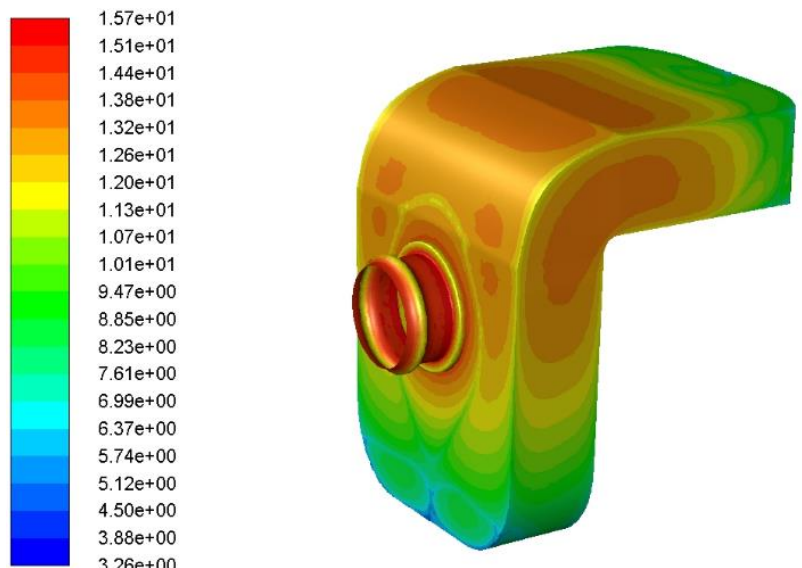

Fig. 7. Shape Sensitivity Magnitude

The result of deformation of the computing area was evaluated in the next step. The unmodified and modified geometry were compared. A Figure 8 shows deformed areas. As show a Figure 8 the input part of the connector tends to expand (detail 1), and slight rounding also occurs on the upper surface of the connector body (detail 2).
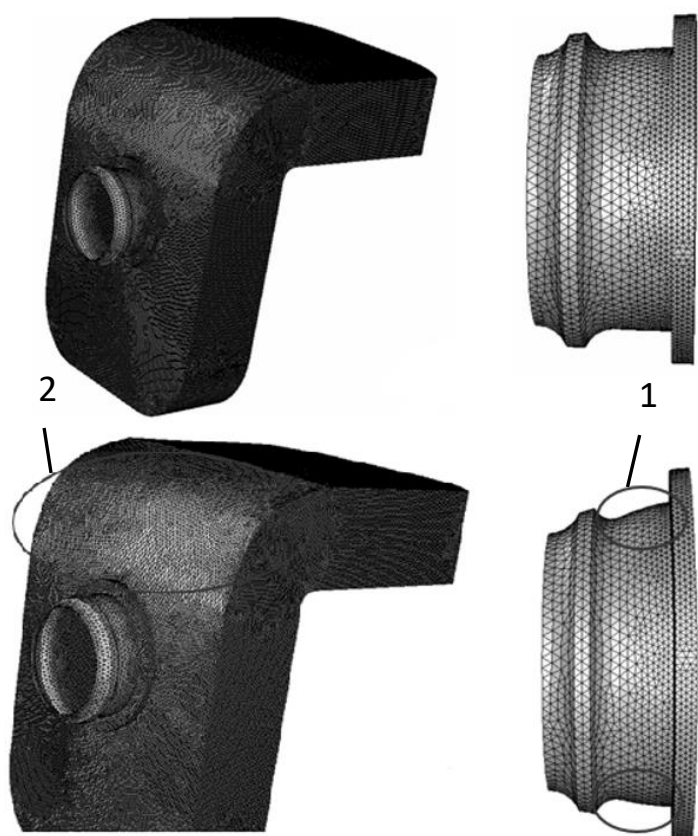

1

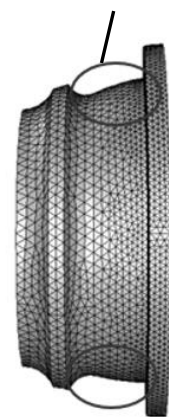

Fig. 8. Deformation: unmodified geometry (Top), modified geometry (Bottom)

A calculation of the basic flow field on the modified geometry was performed and a pressure drop between inlet and outlet $\left(\Delta p_{I N, O U T}=\Delta p_{I N}-\Delta p_{O U T}\right)$ was evaluated. There were 5 optimizations performed. In the Table 4 there is a comparison of $\Delta p_{I N, O U T}$ between original geometry and geometry after optimization.

Table 4. Evaluation of $\Delta p_{I N, O U T}(\eta=1 \mathrm{~Pa} \cdot \mathrm{s})$, percentage decrease

\begin{tabular}{|l|c|}
\hline & $\Delta p_{\text {IN, OUT }}[\mathbf{P a}]$ \\
\hline Unmodified geometry & 46719.45 \\
\hline Modified geometry & 45003.41 \\
\hline Decrease [\% ] & $\mathbf{3 . 6 7}$ \\
\hline
\end{tabular}


As can be seen from the Table 4, the pressure drop between inlet and outlet for a changed dynamic viscosity dropped by $3.67 \%$.

In the last step, a calculation of the basic flow field for the modified geometry was performed. The dynamic viscosity was defined to the original value $(0.0019 \mathrm{~Pa} \cdot \mathrm{s})$.

The pressure drop between inlet and outlet $\triangle p_{I N, O U T}$ was evaluated again and compared with $\Delta p_{I N, O U T}$ for the original unmodified geometry.

Table. 5. Evaluation of $\Delta p_{I N, O U T}(\eta=0.0019 \mathrm{~Pa} \cdot \mathrm{s})$, percentage

\begin{tabular}{|l|c|}
\hline \multicolumn{2}{|c|}{ decrease } \\
\hline Unmodified geometry & Ap IN,out $[\mathbf{P a}]$ \\
\hline Modified geometry & 3186.6 \\
\hline Decrease [\%] $]$ & 2425.3 \\
\hline
\end{tabular}

The pressure drop between inlet and outlet after a recalculation with the original viscosity dropped by $23.9 \%$, as shown in the Table 5 .

The character of the flow field was evaluated by a vector field in longitudinal section of the computing area with original viscosity (0.0019 Pas) for unmodified geometry (Figure 9) and for modified geometry (Figure 10).
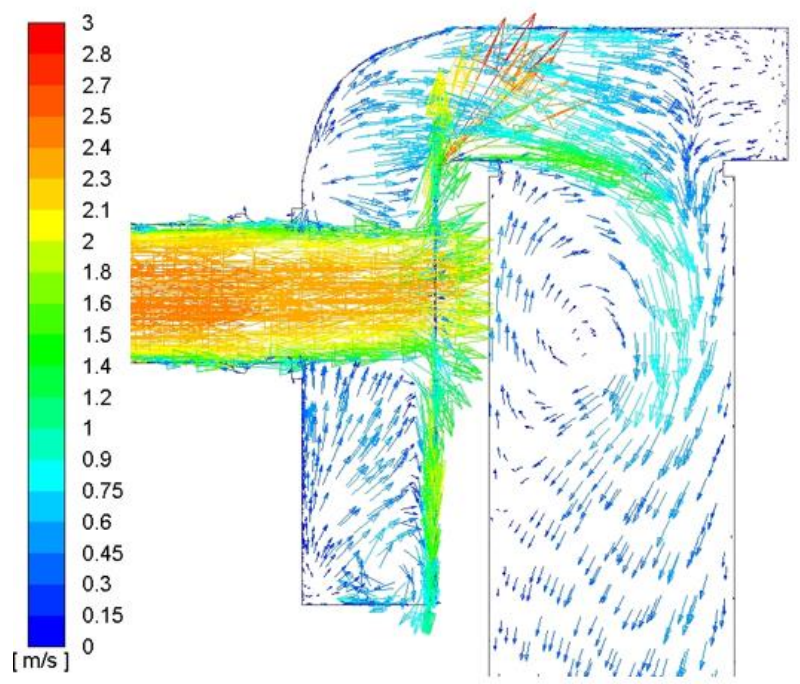

Fig. 9. Velocity Vectors Colored by Velocity

Magnitude $v / \mathrm{m} / \mathrm{s}$ : unmodified geometry

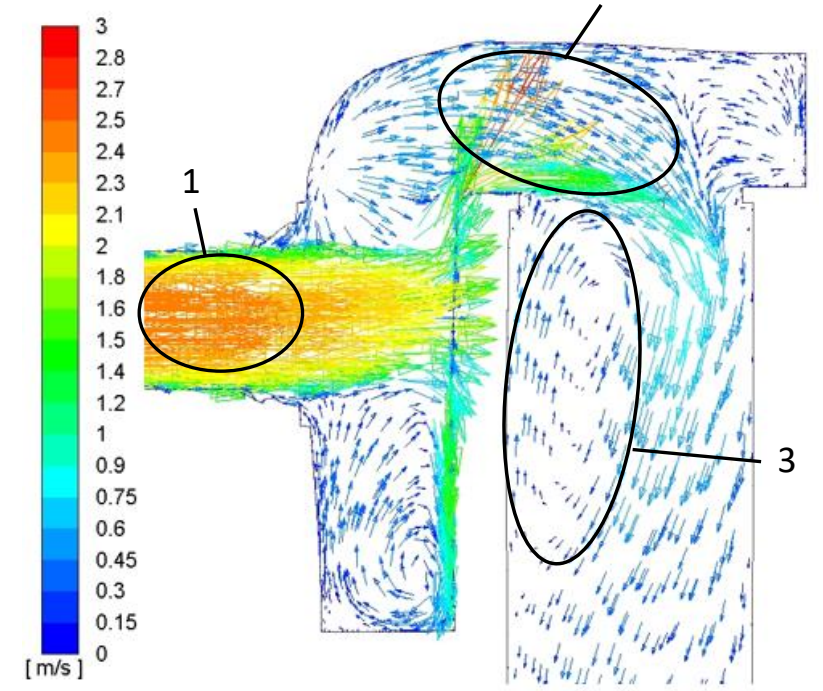

Fig. 10. Velocity Vectors Colored by Velocity Magnitude $v / \mathrm{m} / \mathrm{s}$ : modified geometry

The vector fields for both variants were evaluated in the same range. It is obvious a higher maximum speed in the inlet mid-section (detail 1), as shown in Figure 10. There is a decrease in speed (detail 2) in the transition section of the outlet pipe. There is also a different type of vortex in the output pipe. The vortex is extended more to the wall (detail 3).

The contours of total pressure for unmodified geometry (Figure 11) and for modified geometry (Figure 12) with the original viscosity in the longitudinal section of the computing area were evaluated. These both variants were evaluated in the same range. The modification will reduce the total pressure in the input part of the computing area (detail 4) compared to the unmodified geometry (Figure 11).

An analogous result with the vector field is apparent. The velocity has increased in this area, i.e. the total pressure drops. This result is also evident by the pressure drop $\Delta p_{I N, O U T}$ evaluation.

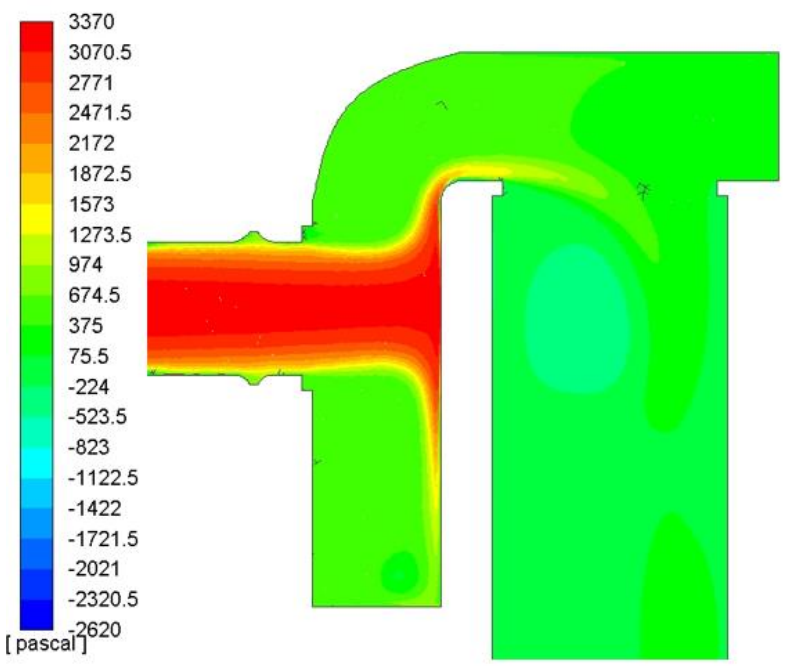

Fig. 11. Contours of Total Pressure $p / \mathrm{Pa}$ : unmodified geometry 


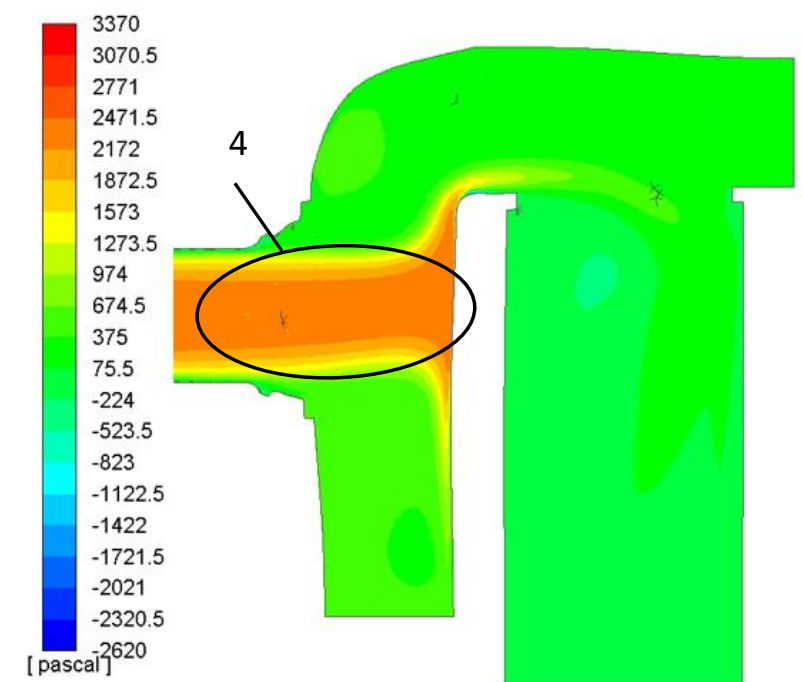

Fig. 12. Contours of Total Pressure $p$ / Pa: modified geometry

\section{Conclusion}

This paper deals with the methodology of using the Adjoint Solver optimization tool to minimize pressure drop in ANSYS Fluent software. The basic flow was calculated on the intercooler entry (cold) connector geometry in the first phase. The coolant was water-glycol in ratio 60/40. Several mathematical models of turbulence have been tested. It was used $k-\varepsilon$ RNG Enhanced Wall Treatment mathematical model of turbulence for evaluation. By evaluating parameter $y+$ was verified that this mathematical model of turbulence is used correctly.

The shape optimization in Adjoint Solver was performed on the entry (cold) connector geometry (Figure 2). There are vortexes in the computing area and neither the use of stabilization schemes has not led to a convergent solution (Figure 6). The dynamic viscosity of coolant was adjusted to $1 \mathrm{~Pa}$ s to avoid formation of vortices. This provides stable and convergent calculation of adjoint variables. Then the optimization was performed. The deformation region was defined only on the inlet part of the entry connector as shown in Figure 7 . The optimization was performed according to a mentioned methodology. The result of deformation of the computing area was evaluated in the next step. As show a Figure 8 the input part of the connector tends to expand, and slight rounding also occurs on the upper surface of the connector body.

A calculation of the basic flow field on the modified geometry was performed with changed dynamic viscosity $(\eta=1 \mathrm{~Pa} \cdot \mathrm{s})$ and a pressure drop between inlet and outlet $\triangle p_{\text {IN,OUT }}$ was evaluated. As can be seen from the Table 4, the pressure drop between inlet and outlet for the changed dynamic viscosity dropped by $3.67 \%$. In the last step, a calculation of the basic flow field for the modified geometry was performed. The dynamic viscosity was defined to the original value (0.0019 Pas). The pressure drop between inlet and outlet $\Delta p_{I N, O U T}$ was evaluated again. The pressure drop between inlet and outlet after a recalculation with the original viscosity dropped by $23.9 \%$, as shown in the Table 5 .
This paper was supported by the Project No. CZ.02.1.01/0.0/0.0/16 019/0000867 „European Regional Development Fund in the Research Centre of Advanced Mechatronic Systems project" within the Operational Programme Research, Development and Education.

\section{References}

1. A. Tzanakis, Duct optimization using CFD software 'ANSYS Fluent Adjoint Solver'. Chalmers University of Technology in Göteborg, pp. 42, (2014)

2. M. Kozubková, M. Bojko, L. Hružík, 37th Meeting of Departments of Fluid Mechanics and Thermodynamics, Investigation of flow for check valves using the optimization method, 2000 (2018).

3. R. Sikora, A. Bureček, L. Hružík, M. Vašina, EPJ Web of Conferences, EFM14 Experimental investigation of cavitation in pump inlet, 92, 02081, (2015)

4. M. Kozubková, Modelováni prouděni tekutin: FLUENT, CFX, VŠB-TUO, pp. 153, (2008)

5. M. Kozubková, T. Blejchař, M. Bojko, Modelování prenosu tepla, hmoty a hybnosti, VŠB-TUO, pp. 174, (2011)

6. ANSYS FLUENT Manual. ANSYS FLUENT Advanced Add-On Modules Version 16.2. ANSYS, Inc., pp. 474, (2015)

7. M. Kozubková, M. Bojko, Aplikace adjungovaného řšiče teorie, VŠB-TUO, (2015) 